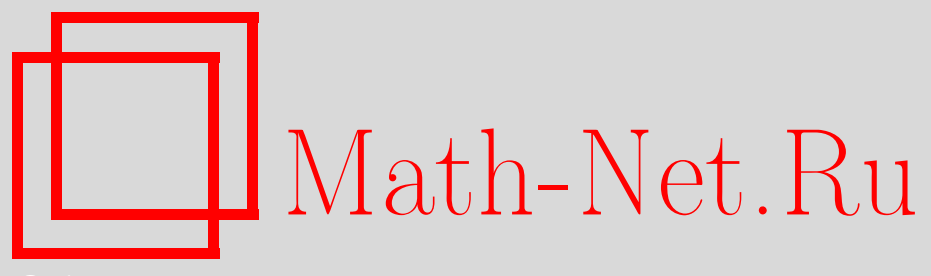

Б. Фидлер, К. Гротта-Рагаццо, К. Роша, Явный вид функции Ляпунова для зеркально симметричных параболических уравнений в частных производных на окружности, УМH, 2014, том 69, выпуск 3, 27-42

DOI: https://doi.org/10.4213/rm9591

Использование Общероссийского математического портала Math-Net.Ru подразумевает, что вы прочитали и согласны с пользовательским соглашением http://www . mathnet.ru/rus/agreement

Параметры загрузки:

IP : 54.196 .121 .252

26 апреля 2023 г., 15:16:58

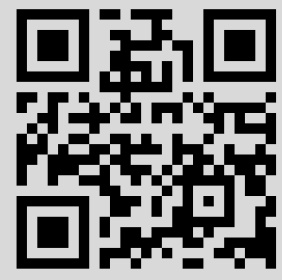




\section{Явный вид функции Ляпунова для зеркально симметричных параболических уравнений в частных производных на окружности}

\section{Б. Фидлер, К. Гротта-Рагаццо, К. Роша}

В работе в явном виде строится функция Ляпунова для скалярных параболических уравнений типа "реакция-адвекция-диффузия" с периодическими граничными условиями. Предполагается, что нелинейность является четной по адвективному члену. Мы следуем оригинальному подходу Х. Матано, предложенному (и пригодному только) для разделенных граничных условий.

Библиография: 20 названий.

Ключевые слова: дифференциальные уравнения с частными производными, вариационные методы, функционал энергии, конвекция, адвекция, уравнения реакции-диффузии, периодические граничные условия.

DOI: $10.4213 / \mathrm{rm} 9591$

\section{СОДЕРЖАНИЕ}

1. Введение и формулировка основного результата................ 27

2. Конструкция Матано.................................... 33

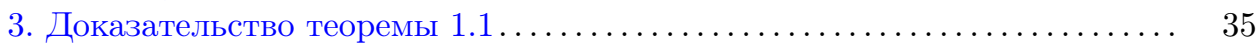

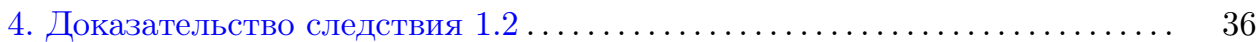

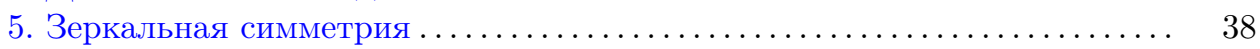

6. Заключительные комментарии . . . . . . . . . . . . . . . . . . . . 39

Список литературы ........................................... 41

\section{1. Введение и формулировка основного результата}

Мы рассматриваем действительные скалярные полулинейные параболические уравнения в частных производных вида

$$
u_{t}=u_{x x}+f,
$$

где $x$ - пространственная координата, $0<x<1$, и $f$ - нелинейность класса $C^{1}$.

Работа Б. Фидлера и К. Роша выполнена при поддержке Deutsche Forschungsgemeinschaft, ФРГ (проект SFB 647 Space Time Matter) и Fundaçåo para a Ciência e a Tecnologia, Португалия. Работа K. Гротта-Рагаццо выполнена при частичной поддержке Conselho Nacional de Desenvolvimento Cientifico e Tecnológíco, Бразилия (проект № 305089/2009-9).

(C) Б. ФИДлЕР, К. ГРОтТА-РАГАЦцО, К. РОшА, 2014 
Следуя совету М. И. Вишика "nicht zu eilen”, мы в данной работе остановимся лишь на вопросе существования и несуществования функций Ляпунова. Это один из ключевых вопросов, возникающих в наших продолжающихся исследованиях характера и структуры динамики на глобальных аттракторах параболических уравнений (1.1), которые рассматриваются в деталях в простейшей скалярной форме (см., например, [7], [8] и приведенную там библиографию). На глобальном уровне, наши работы являются лишь скромной попыткой исследования малой части всего богатства глобальных аттракторов уравнений в частных производных, раскрываемого, к примеру, в фундаментальной монографии А.В. Бабина и М.И. Вишика [3] и в работах их многочисленных последователей по всему миру.

В случае разделенных граничных условий Дирихле или Неймана

$$
u=0 \quad \text { или } \quad u_{x}=0
$$

при $x=0,1$ и нелинейностей вида

$$
f=f(x, u)
$$

хорошо известно, что функцию Ляпунова можно выписать в явном виде

$$
V(u):=\int_{0}^{1} L\left(x, u, u_{x}\right) d x
$$

с лагранжианом

$$
L\left(x, u, u_{x}\right):=\frac{1}{2} u_{x}^{2}-F(x, u) .
$$

Здесь $F$ - первообразная функции $f$ по $u$. Функция Ляпунова $V$ удовлетворяет уравнению

$$
\dot{V}=\frac{d}{d t} V(u(t, \cdot))=-\int_{0}^{1}\left(u_{t}\right)^{2} d x
$$

вдоль любого классического решения $u=u(t, x)$ уравнения (1.1). С учетом принципа инвариантности Ла-Салля отсюда вытекает сходимость к равновесному состоянию ограниченных решений при $t \rightarrow+\infty$. Путем добавления подходящих граничных членов к функции Ляпунова $V$ данный результат можно перенести на разделенные нелинейные граничные условия

$$
u_{x}=\beta(x, u), \quad x=0,1,
$$

типа Робена. Аналогичные утверждения остаются верными при переходе от сильных решений к слабым. При этом соответствующий полупоток, определяемый (1.1), оказывается $L^{2}$-градиентным полупотоком функции Ляпунова $V$. Основы общей теории содержатся в книгах [10] и [15]; по поводу нелинейностей класса $C^{1}$ мы отсылаем читателя к статье [13].

Несколько менее известным является то, как Т.И. Зеленяк [19] и затем Х. Матано [11] обобщили этот классический результат на нелинейности

$$
f=f\left(x, u, u_{x}\right)
$$


зависящие также от адвективного члена $u_{x}$ (снова при разделенных граничных условиях). Для удобства читателя в разделе 2 мы напомним красивое рассуждение, принадлежащее Матано [11]. После подстановки подходящего лагранжиана $L=L(x, u, p)$ в (1.5) свойство убывания по Ляпунову (1.6) заменяется на следующее:

$$
\dot{V}=\frac{d}{d t} V(u(t, \cdot))=-\int_{0}^{1} L_{p p}\left(x, u, u_{x}\right)\left(u_{t}\right)^{2} d x ;
$$

при этом функция $p \mapsto L(x, u, p)$ строго выпукла, т. е. вторая частная производная по $p$ положительна:

$$
L_{p p}>0
$$

Как следствие, $L_{p p}$ можно рассматривать как неоднородную $L^{2}$-метрику, относительно которой задача (1.1), (1.2), (1.8) определяет градиентный полупоток.

При периодических граничных условиях $x \in S^{1}:=\mathbb{R} / \mathbb{Z}$, т. е.

$$
[u]_{0}^{1}=\left[u_{x}\right]_{0}^{1}=0,
$$

параболическое уравнение (1.1) сохраняет градиентный характер (1.1)-(1.6) для нелинейностей $f=f(x, u)$. Однако из-за присутствия адвективного члена $u_{x}$ возможно появление неравновесных периодических по времени решений $u(t, x)$. Например, для $\mathrm{SO}(2)$-эквивариантного случая

$$
f=f\left(u, u_{x}\right)
$$

функции $u(t, x)$ и $u(t, x+\vartheta)$ одновременно являются решениями уравнения $(1.1)$ для любого фиксированного вращения $\vartheta \in S^{1}=\mathrm{SO}(2)$. Еще в работе [2] была отмечена возможность существования пространственно-неоднородных решений в виде вращающихся волн

$$
u=U(x-c t)
$$

с ненулевыми волновыми скоростями $c \neq 0$. В самом деле, для этого достаточно всего лишь потребовать существование нестационарных 1-периодических решений $U$ уравнения бегущей волны

$$
U^{\prime \prime}+c U^{\prime}+f\left(U, U^{\prime}\right)=0
$$

В общем случае к сходимости к равновесному состоянию при $t \rightarrow+\infty$ добавляется возможная сходимость к вращающимся волнам. В качестве конкретного примера рассмотрим нелинейность

$$
f(u, p):=\lambda u\left(1-u^{2}\right)-c p,
$$

где $\lambda>\pi$. Нам достаточно рассмотреть решения с кубической нелинейностью

$$
f_{0}(u):=\lambda u\left(1-u^{2}\right)
$$

(в этом случае (1.1) является задачей Чэфи-Инфанте; см. [4]) в координатах, вращающихся с постоянной скоростью $c$ вокруг $x \in S^{1}$. Тогда неоднородное 
равновесное состояние $U(x)$ задачи Чэфи-Инфанте (1.1), (1.16) дает неоднородное решение в виде вращающихся волн $U(x-c t)$ уравнения (1.14). Конечно, данный метод можно распространить на произвольные нелинейности вида $f(u, p)=f_{0}(u)-c p$. Другие примеры включают в себя нелинейности вида $f=f(u, p)$ для уравнения бегущей волны (1.14) типа Ван дер Поля. Для общих, не обязательно $\mathrm{SO}(2)$-эквивариантных нелинейностей $f=f(x, u, p)$ могут возникать периодические по времени решения $u=u(t, x)$, не являющиеся вращающимися волнами. Тем не менее остается верной теорема Пуанкаре-Бендиксона о существовании дихотомии между равновесными и периодическими решениями при $t \rightarrow+\infty$ (см. [5]).

После этих пояснений мы перейдем к рассмотрению $\mathrm{O}(2)$-эквивариантного случая уравнения (1.1) с периодическими граничными условиями (1.11). Чтобы учесть симметричные отражения $x \mapsto-x \in S^{1}$, мы предположим, что нелинейность $f$ является четной по $p=u_{x}$, а именно,

$$
f=f(u, p):=\bar{f}\left(u, \frac{1}{2} p^{2}\right),
$$

где нелинейность

$$
\bar{f}=\bar{f}(u, q), \quad q=\frac{1}{2} p^{2},
$$

принадлежит классу $C^{1}$. Далее, с использованием аргументов, основанных на свойствах Штурма узловых точек, т. е. нулей и точек смены знака (zero numbers [2], [5]), можно показать, что все вращающиеся волны замораживаются, т. е. "вращаются" со скоростью $c=0$, оказываясь положениями равновесия (см. также [7]). Вместо этого для $\mathrm{O}(2)$-случая мы в явном виде строим функцию Ляпунова, что позволяет вывести сходимость к равновесному состоянию непосредственно из принципа инвариантности Ла-Салля. В этом случае сходимость к единственному равновесному состоянию была установлена еще Матано [11]. Однако в его подходе, основанном на свойствах Штурма и не использующем явной функции Ляпунова, фактически исключалась альтернатива вращающихся волн. Ниже мы построим явную функцию Ляпунова для исследования вариационного характера уравнения (1.1) на окружности, порождающего градиентный полупоток.

Для формулировки основного результата работы (теоремы 1.1) мы предположим, что $\mathrm{O}(2)$-эквивариантная нелинейность $f=\bar{f}(u, q)$ (см. $(1.17),(1.18))$ такова, что неавтономное ОДУ

$$
\begin{aligned}
\frac{d}{d u} q & =-\bar{f}(u, q), \\
q\left(u_{0}\right) & =q_{0},
\end{aligned}
$$

имеет глобальное решение

$$
q\left(u_{1}\right)=\Psi^{u_{1}, u_{0}}\left(q_{0}\right)
$$

при действительных $q_{0}, u_{0}, u_{1}$. Это требование удовлетворяется, если $\bar{f}$ растет не быстрее чем линейно по $q$. K примеру, одностороннее ограничение 
типа $u \bar{f}(u, q) \leqslant c_{1}(u)+c_{2}(u) q$ в интересующем нас интервале $q \geqslant 0$ при непрерывных $c_{1}, c_{2}$ препятствует разрушению решений уравнения (1.19) при конечных "временах" $u$ (см. также $[16 ; \S 2])$.

Определим лагранжиан $L$ (подынтегральное выражение функции Ляпунова $V$, заданной формулой (1.4)) следующим образом:

$$
L(u, p):=\int_{0}^{p} \int_{0}^{p_{1}} \exp \left(F_{q}\left(u, \frac{1}{2} p_{2}^{2}\right)\right) d p_{2} d p_{1}-F(u),
$$

где

$$
\begin{aligned}
F(u) & :=\int_{0}^{u} \bar{f}\left(u_{1}, 0\right) \exp \left(F_{q}\left(u_{1}, 0\right)\right) d u_{1}, \\
F_{q}(u, q) & :=\int_{0}^{u} \bar{f}_{q}\left(u_{1}, \Psi^{u_{1}, u}(q)\right) d u_{1} .
\end{aligned}
$$

Здесь $\bar{f}_{q}=\bar{f}_{q}\left(u_{1}, q_{1}\right)$ обозначает частную производную по второму аргументу $q_{1}=\Psi^{u_{1}, u}(q)$, а не полную производную сложной функции $q \mapsto \bar{f}_{q}\left(u_{1}, \Psi^{u_{1}, u}(q)\right)$ по $q$.

ТеОРема 1.1. Предположим, что для $\bar{f} \in C^{1}$ решение (1.20) ОДУ (1.19) существует глобально.

Тогда функиионал

$$
V(u):=\int_{0}^{1} L\left(u, u_{x}\right) d x
$$

с лагранжианом L, заданным формулой (1.21), является функиией Ляпунова параболического уравнения (1.1) с $\mathrm{O}(2)$-эквивариантной нелинейностъю $f=$ $\bar{f}\left(u, u_{x}^{2} / 2\right)$ при периодических граничных условиях (1.11). Точнее говоря, равенство

$$
\dot{V}=\frac{d}{d t} V(u(t, \cdot))=-\int_{0}^{1} L_{p p}\left(u, \frac{1}{2}\left(u_{x}\right)^{2}\right)\left(u_{t}\right)^{2} d x
$$

имеет место для классических решений $u=u(t, x)$ задачи (1.1), причем лагранжиан $L(x, u, p)$ является строго выпуклым по $p$, m.е. метрический коэффициент

$$
L_{p p}=\exp \left(-F_{q}\left(u, \frac{1}{2} p^{2}\right)\right)
$$

положителен.

Если $\bar{f}\left(u, u_{x}^{2} / 2\right)=f(u)$ не зависит от $q=p^{2} / 2=u_{x}^{2} / 2$, то $\bar{f}_{q} \equiv 0, F_{q} \equiv 0$, $L_{p p} \equiv 1$ и $L(u, p)=p^{2} / 2-F(u)$ с первообразной функцией $F^{\prime}=f$, что дает классическую функцию Ляпунова (1.4)-(1.6) в теореме 1.1.

В определении (1.21), (1.22) лагранжиана $L(u, p)$ по-прежнему участвуют повторные интегралы, в которые входят эволюция $\Psi^{u_{1}, u_{0}}\left(q_{0}\right)$ характеристического ОДУ $(1.19),(1.20)$ и нелинейность $\bar{f}$. Чтобы избавиться от части интегралов и получить более простое выражение для $L$, мы рассмотрим вспомогательную функцию $\varphi=\varphi(u, p)$ со свойством

$$
\begin{aligned}
\varphi_{p}(u, p) & =\Psi_{q}^{0, u}\left(\frac{1}{2} p^{2}\right), \\
\varphi(u, 0) & =0 .
\end{aligned}
$$


Здесь $\Psi_{q}^{0, u}(q)$ обозначает частную производную эволюции $\Psi^{0, u}(q)$ по $q$. Ясно, что такая $\varphi(u, p)$ получается простым интегрированием первого уравнения B $(1.26)$ :

$$
\varphi(u, p):=\int_{0}^{p} \Psi_{q}^{0, u}\left(\frac{1}{2} p_{2}^{2}\right) d p_{2} .
$$

СЛЕДСТвИЕ 1.2. Лагранжиан L, определенный в (1.21), (1.22), можно представить в эквивалентном виде

$$
L(u, p)=p \varphi(u, p)-\Psi^{0, u}\left(\frac{1}{2} p^{2}\right)
$$

Как и выше, $L(u, p)=p^{2}-p^{2} / 2-F(u)=p^{2} / 2-F(u)$ в тривиальном случае $\bar{f}_{q}=F_{q}=0, \Psi^{0, u}(q)=q+F(u), \Psi_{q}^{0, u}(q)=1, \varphi(u, p)=p$. Явное построение функции Ляпунова $V$ также возможно, если $f(u, p)=a(u)+b(u) p^{2} / 2$. В этом случае уравнение (1.19) становится линейным и может быть проинтегрировано. После преобразований мы можем выразить лагранжиан $L$ функции $V$ через интегралы от функций $a$ и $b$. При линейной функции $a(u)$ и постоянной $b$ явное выражение функции Ляпунова было также получено в предложении 5.8 работы [9] с использованием идей из гл. 2 монографии [20].

В разделе 2 мы приводим элегантную конструкцию Матано лагранжиана при $f=f\left(x, u, u_{x}\right)$ и указываем места, в которых, как и следовало ожидать, рассуждение теряет силу на техническом уровне при периодических граничных условиях. В разделе 3 мы доказываем теорему 1.1, отталкиваясь от конструкции Матано. Альтернативный подход заключается в том, что при выполнении условия (1.17) уравнение равновесия $u_{x x}+f=0$ имеет первый интеграл (см. [16]). В разделе 4 мы доказываем следствие 1.2. Далее, в разделе 5 приводится пример, показывающий, что функция Ляпунова может не существовать (как и следовало ожидать) на окружности $x \in S^{1}$ для нелинейностей $f(x, u, p)=f(-x, u,-p)$, которые обладают лишь зеркальной симметрией, а не полной $\mathrm{O}(2)$-эквивариантностью: $f(u, p)=f(u,-p)$, т. е. $f=\bar{f}\left(u, p^{2} / 2\right)$. Как и выше, это происходит из-за наличия нестационарных периодических по времени орбит. В разделе 6 мы кратко останавливаемся на связанных с нашими вопросами задачах о глобальных аттракторах уравнений в частных производных, квазилинейных уравнениях, а также на случае отрицательных $q=u_{x}^{2} / 2$, т. е. комплексных $u_{x}$.

Б. Фидлер выражает глубокую признательность Х. Матано, который много лет назад обратил внимание автора на свою конструкцию лагранжиана для нелинейностей $f=f\left(x, u, u_{x}\right)$ при разделенных граничных условиях, а также прокомментировал ее. Авторы выражают благодарность А.Р. Алимову и П.Л. Гуревичу за работу над переводом и замечания, способствовавшие улучшению статьи. Авторы также выражают признательность М. Баретт (M. Barrett) за помощь в наборе текста статьи. 


\section{2. Конструкция Матано}

В данном разделе мы напоминаем конструкцию Матано [11] лагранжиана $L=L(x, u, p)$, для которого

$$
V(u):=\int_{0}^{1} L\left(x, u, u_{x}\right) d x
$$

является функцией Ляпунова для уравнения в частных производных (1.1) с разделенными граничными условиями Дирихле или Неймана (1.2) в случае нелинейностей общего вида $f=f\left(x, u, u_{x}\right)$. Мы показываем, что

$$
\dot{V}=\frac{d}{d t} V(u(t, \cdot))=-\int_{0}^{1} L_{p p}\left(x, u, u_{x}\right) \cdot\left(u_{t}\right)^{2} d x
$$

при условии, что функция $p \mapsto L(x, u, p)$ строго выпукла, т. е. вторая частная производная по $p$ положительна:

$$
L_{p p}>0
$$

По поводу сходной оригинальной конструкции Зеленяка мы отсылаем читателя к лемме 1 работы [19]. Двадцатью годами позже в работе Матано [11; приложение] эта оригинальная конструкция была вызвана из забвения, разъяснена и незначительно модифицирована при менее ограничительных предположениях. Различия становятся более очевидными в более общем квазилинейном параболическом случае (6.1), на котором мы кратко остановимся в разделе 6 . Для ясности изложения мы будем следовать подходу Матано, а не оригинальным идеям Зеленяка, которые недостаточно прозрачны. Стоит еще раз подчеркнуть, что обе конструкции функции Ляпунова ограничиваются разделенными граничными условиями, хотя и несколько более общего вида, и не работают для периодических граничных условий.

Конструкция такова. Для классических решений $u=u(t, x)$ мы интегрируем (2.1) по частям и подставляем в получившееся выражение $u_{x x}=u_{t}-f$. С учетом (1.1) это дает требуемый результат:

$$
\begin{aligned}
\dot{V} & =\int_{0}^{1}\left(L_{u} u_{t}+L_{p} u_{t x}\right) d x \\
& =\int_{0}^{1}\left(L_{u}-\frac{d}{d x} L_{p}\left(x, u, u_{x}\right)\right) u_{t} d x \\
& =\int\left(L_{u}-L_{p x}-L_{p u} u_{x}-L_{p p} u_{x x}\right) u_{t} d x \\
& =\int_{0}^{1}\left(\left(L_{u}-L_{p x}-L_{p u} u_{x}+L_{p p} f\right) u_{t}-L_{p p}\left(u_{t}\right)^{2}\right) d x \\
& =-\int_{0}^{1} L_{p p}\left(u_{t}\right)^{2} d x .
\end{aligned}
$$

Здесь мы для простоты предположили, что выполнены граничные условия Дирихле $u=0$, и, значит, $u_{t}=0$ при $x=0,1$. Путем добавления подходящих 
граничных членов к функции Ляпунова $V$ данный результат можно перенести на граничные условия Неймана или более общие нелинейные граничные условия (1.7) типа Робена. Естественно, для выполнения последнего равенства лагранжиан $L$ должен удовлетворять линейному уравнению в частных производных первого порядка

$$
L_{u}-L_{x p}-p L_{u p}+f L_{p p}=0
$$

при всех действительных $u, p$ и $0 \leqslant x \leqslant 1$. Х. Матано применяет анзац

$$
L_{p p}=: \exp (g)
$$

для понижения порядка уравнения и обеспечения выполнения условия выпуклости $L_{p p}>0$.

При дифференцировании уравнения (2.5) по $p$ члены $L_{u p}$ взаимно сокращаются и мы приходим к линейному уравнению в частных производных первого порядка по $g=g(x, u, p)$ :

$$
g_{x}+p g_{u}-f g_{p}=f_{p},
$$

которое может быть решено методом характеристик: вдоль решений $(u, p)(x)$ ОДУ

$$
\begin{aligned}
& \frac{d u}{d x}=p, \\
& \frac{d p}{d x}=-f(x, u, p)
\end{aligned}
$$

функция $x \mapsto g=g(x, u(x), p(x))$ должна иметь полную производную

$$
\frac{d}{d x} g=f_{p}(x, u, p)
$$

Отсюда, предположив, например, что

$$
g(0, u, p) \equiv 0,
$$

мы получим $g$ глобально, при условии, что решения характеристического ОДУ (2.8) существуют при всех $0 \leqslant x \leqslant 1$ и всех действительных начальных условиях $u, p$ в $x=0$. Иными словами, переходя от $(2.7) \cdot L_{p p}=(2.5)_{p}$ к (2.5) с учетом (2.6), мы положим

$$
\begin{aligned}
L(x, u, p) & :=\int_{0}^{p} \int_{0}^{p_{1}} \exp \left(g\left(x, u, p_{2}\right)\right) d p_{2} d p_{1}-F(x, u), \\
F(x, u) & :=\int_{0}^{u} f\left(x, u_{1}, 0\right) \exp \left(g\left(x, u_{1}, 0\right)\right) d u_{1} .
\end{aligned}
$$

В самом деле, по построению левая часть (2.5) не зависит от $p$. Как следствие, (2.5) будет выполнено при всех $p$, если мы проверим, что (2.5) имеет место при $p=0$. При $p=0$ из определения (2.11) вытекает, что $0 \equiv L_{p} \equiv L_{p x}$ и $L_{u}=-F_{u}=-f \exp (g)=-f L_{p p}$. Это доказывает $(2.5)$, завершая конструкцию Матано функции Ляпунова $V$. 
Вполне понятно, что данная корректная конструкция может стать неприменимой при наложении периодических граничных условий. Это на самом деле и происходит. Предположим, что характеристическое уравнение $(2.8)$ имеет 1-периодическую орбиту $(u, p)(x)$, т. е.

$$
[(u, p)(x)]_{0}^{1}=0 .
$$

С учетом (2.9) из 1-периодичности отображения $x \mapsto g(x, u, p)$ следует, что

$$
\begin{aligned}
0 & =[g(x, u(0), p(0))]_{0}^{1}=[g(x, u(x), p(x))]_{0}^{1} \\
& =\int_{0}^{1} \frac{d}{d x} g(x, u(x), p(x)) d x=\int_{0}^{1} f_{p}(x, u(x), p(x)) d x .
\end{aligned}
$$

Но это условие интегрируемости для $f_{p}$ легко нарушить, оставляя периодическую орбиту $(u, p)(x)$ без изменения. Таким образом, конструкция Матано перестает быть верной в общем случае при наличии периодических по времени орбит в уравнении (1.1). Нелокальность некорректного условия совместности

$$
[g(x, u(x), p(x))]_{0}^{1}=\int_{0}^{1} f_{p}(x, u(x), p(x)) d x
$$

вдоль характеристик (2.8) может быть использована для модификации конструкции Матано в $\mathrm{O}(2)$-эквивариантном случае.

\section{3. Доказательство теоремы 1.1}

Идея доказательства теоремы 1.1 состоит в небольшом видоизменении конструкции Матано из раздела 2 на случай $\mathrm{O}(2)$-эквивариантных нелинейностей

$$
f=f(u, p)=\bar{f}(u, q), \quad q:=\frac{1}{2} p^{2} .
$$

В силу зеркальной инвариантности нелинейность $f$ является четной по $p=u_{x}$, а из-за инвариантности относительно поворотов она не зависит явно от $x$. Как следствие, мы рассмотрим не зависящий от $x$ лагранжиан $L=L(u, p)$ и будем искать $\mathrm{O}(2)$-инвариантные функции Ляпунова вида

$$
V(u):=\int_{0}^{1} L\left(u, u_{x}\right) d x,
$$

где

$$
L_{p p}=\exp (g)>0
$$

a $g$ зеркально симметрична:

$$
g=g(u, p)=\bar{g}(u, q), \quad q:=\frac{1}{2} p^{2} .
$$

Повторяя рассуждения Матано (2.4)-(2.7), мы приходим к линейному уравнению в частных производных первого порядка

$$
p\left(\bar{g}_{u}-\bar{f} \bar{g}_{q}\right)=p \bar{f}_{q} .
$$


Здесь мы воспользовались правилом дифференцирования сложной функции и подставили в (2.7) определения (3.1), (3.4) функций $f, g$. Далее, поделив на $p$, мы решаем уравнение

$$
\bar{g}_{u}-\bar{f} \bar{g}_{q}=\bar{f}_{q}
$$

методом характеристик вдоль глобальных решений $q\left(u_{1}\right)=\Psi^{u_{1}, u_{0}}\left(q_{0}\right)$ уравнения

$$
\begin{aligned}
\frac{d}{d u} q & =-\bar{f}(u, q), \\
q\left(u_{0}\right) & =q_{0},
\end{aligned}
$$

определенных в (1.19), (1.20). Тогда линейное уравнение в частных производных первого порядка (3.5) выполнено для любой $\bar{g}$, удовлетворяющей уравнению

$$
\frac{d}{d u} \bar{g}(u, q(u))=\bar{f}_{q}(u, q(u))
$$

вдоль характеристик, скажем, с начальным условием

$$
\bar{g}(0, q):=0 .
$$

Рассматривая эволюцию $q\left(u_{1}\right)=\Psi^{u_{1}, u_{0}}\left(q_{0}\right)$, отсюда получаем, что

$$
\bar{g}(u, q)=\int_{0}^{u} \bar{f}_{q}\left(u_{1}, \Psi^{u_{1}, u}(q)\right) d u_{1}=F_{q}(u, q),
$$

где $F_{q}(u, q)$ определена в (1.22).

Как и выше, мы перейдем от (3.10)) к уравнению (2.5), которое теперь принимает вид

$$
L_{u}-p L_{u p}+f L_{p p}=0 .
$$

Полагая $L_{p p}:=\exp (\bar{g})=\exp \left(F_{q}\right)$, мы имеем

$$
L(u, p):=\int_{0}^{p} \int_{0}^{p_{1}} \exp \left(F_{q}\left(u, \frac{1}{2} p_{2}^{2}\right)\right) d p_{2} d p_{1}-F(u) .
$$

Здесь $F(u)$ - подходящая постоянная интегрирования. Чтобы определить $F(u)$, нам достаточно подставить $p=0$ в (3.11), что дает

$$
\frac{d}{d u} F(u)=-L_{u}=f L_{p p}=f(u, 0) \exp \left(F_{q}(u, 0)\right) .
$$

Условия (3.12), (3.13) выполнены для лагранжиана $L$, определенного в (1.21), (1.22). Теорема 1.1 доказана.

\section{4. Доказательство следствия $\mathbf{1 . 2}$}

Следствие 1.2 доказывается явным нахождением интегралов в определении $(1.20),(1.21)$ лагранжиана $L(u, p)$. Альтернативный способ доказательства состоит в непосредственной проверке того, что $V(u)=\int_{0}^{1} L\left(u, u_{x}\right) d x$ является функцией Ляпунова. Однако при таком подходе, в отличие от элегантной конструкции Х. Матано, не обеспечивается построение лагранжиана $L$. 
Для вычисления интегралов (1.21), (1.22) сначала отметим, что производная $\eta\left(u_{1}\right)$ эволюции $\Psi^{u_{1}, u_{0}}\left(q_{0}\right)$ характеристического ОДУ $(1.19)$ по начальному условию $q_{0}$,

$$
\eta\left(u_{1}\right):=\Psi_{q}^{u_{1}, u_{0}}\left(q_{0}\right)
$$

удовлетворяет линеаризованному характеристическому уравнению

$$
\begin{aligned}
\frac{d}{d u_{1}} \eta\left(u_{1}\right) & =-\bar{f}_{q}\left(u_{1}, \Psi^{u_{1}, u_{0}}\left(q_{0}\right)\right) \eta\left(u_{1}\right), \\
\eta\left(u_{0}\right) & =1 .
\end{aligned}
$$

Явное интегрирование (4.2) дает

$$
\eta\left(u_{1}\right)=\exp \left(-\int_{u_{0}}^{u_{1}} \bar{f}_{q}\left(u_{2}, \Psi^{u_{2}, u_{0}}\left(q_{0}\right)\right) d u_{2}\right) .
$$

Полагая $u_{0}=u, u_{1}=0, q_{0}=q$, мы получаем из $(1.22)$

$$
\exp \left(F_{q}(u, q)\right)=\eta(0)=\Psi_{q}^{0, u}(q) .
$$

Подставляя (4.4) при $q=p_{2}^{2} / 2$ в определяющий лагранжиан $L(u, p)$ двойной интеграл (1.21) (который мы назовем $L_{1}$ ) и интегрируя по частям, получаем

$$
\begin{aligned}
L_{1}(u, p) & :=\int_{0}^{p} \int_{0}^{p_{1}} \exp \left(F_{q}\left(u, \frac{1}{2} p_{2}^{2}\right)\right) d p_{2} d p_{1} \\
& =\int_{0}^{p} 1 \cdot \int_{0}^{p_{1}} \Psi_{q}^{0, u}\left(\frac{1}{2} p_{2}^{2}\right) d p_{2} d p_{1} \\
& =\left[p_{1} \int_{0}^{p_{1}} \Psi_{q}^{0, u}\left(\frac{1}{2} p_{2}^{2}\right) d p_{2}\right]_{0}^{p}-\int_{0}^{p} p_{1} \Psi_{q}^{0, u}\left(\frac{1}{2} p_{1}^{2}\right) d p_{1} \\
& =p \varphi(u, p)-\int_{0}^{p^{2} / 2} \Psi_{q}^{0, u}(q) d q \\
& =p \varphi(u, p)-\Psi^{0, u}\left(\frac{1}{2} p^{2}\right)+\Psi^{0, u}(0) .
\end{aligned}
$$

Здесь мы воспользовались определением (1.26) вспомогательной функции $\varphi$, подставив $q=p^{2} / 2$ в интеграл.

Для вычисления оставшегося члена $-F(u)$ в $L=L_{1}-F$ (см. (1.21)) мы сначала отметим, что из свойства эволюции $\Psi^{u_{1}, u_{0}}$ следует, что частная производная $\Psi_{u}^{u_{1}, u}(q)$ удовлетворяет уравнению

$$
\begin{aligned}
\Psi_{u}^{u_{2}, u}(q) & =\lim _{\varepsilon \rightarrow 0} \varepsilon^{-1}\left(\Psi^{u_{2}, u+\varepsilon}(q)-\Psi^{u_{2}, u+\varepsilon}\left(\Psi^{u+\varepsilon, u}(q)\right)\right) \\
& =\Psi_{q}^{u_{2}, u}(q) \bar{f}(u, q)
\end{aligned}
$$

что проверяется непосредственным применением правила дифференцирования сложной функции и определения (1.19) эволюции $\Psi$. Отсюда, воспользовав- 
шись $(1.22),(4.4)$ и (4.6) при $u=u_{1}, u_{2}=q=0$, мы получаем, что

$$
\begin{aligned}
-F(u) & =-\int_{0}^{u} \bar{f}\left(u_{1}, 0\right) \exp \left(F_{q}\left(u_{1}, 0\right)\right) d u_{1} \\
& =-\int_{0}^{u} \bar{f}\left(u_{1}, 0\right) \Psi_{q}^{0, u_{1}}(0) d u_{1}=-\int_{0}^{u} \Psi_{u_{1}}^{0, u_{1}}(0) d u_{1} \\
& =-\left[\Psi^{0, u_{1}}(0)\right]_{0}^{u}=-\Psi^{0, u}(0) .
\end{aligned}
$$

Сложение (4.5) и (4.7) дает

$$
L(u, p)=L_{1}(u, p)-F(u)=p \varphi(u, p)-\Psi^{0, u}\left(\frac{1}{2} p^{2}\right),
$$

что и требуется в (1.28). Следствие доказано.

\section{5. Зеркальная симметрия}

В данном разделе мы рассмотрим параболическое уравнение в частных производных (1.1) с периодическими граничными условиями $x \in S^{1}=\mathbb{R} / 2 \pi \mathbb{Z}$ (как в (1.11)). От нелинейности $f=f\left(x, u, u_{x}\right)$ потребуем лишь, чтобы она обладала зеркальной симметрией $x \mapsto-x$, т. е.

$$
f(-x, u,-p)=f(x, u, p) .
$$

В духе старого результата о вложении потоков (см. [17]) мы показываем, что любой двумерный поток

$$
\begin{aligned}
& \dot{a}=g(a, b), \\
& \dot{b}=h(a, b)
\end{aligned}
$$

может быть реализован посредством вложения (5.5) (см. ниже) в классе уравнений в частных производных при условии, что (5.2) обладает зеркальной симметрией, т. е.

$$
\begin{aligned}
& g(a,-b)=g(a, b), \\
& h(a,-b)=-h(a, b) .
\end{aligned}
$$

Поскольку существуют зеркально симметричные двумерные векторные поля с нестационарными периодическими орбитами, уравнение в частных производных (1.1) с соответствующей нелинейностью $f$ не обладает функций Ляпунова вида (1.4), (1.9), (1.10).

Мы реализуем поток ОДУ (5.2) в инвариантном подпространстве

$$
E=\operatorname{span}\{\boldsymbol{c}, \boldsymbol{s}\},
$$

натянутом на первые моды Фурье $\boldsymbol{c}=\cos x, s=\sin x$. Действительно, положим

$$
f(x, a \boldsymbol{c}+b \boldsymbol{s},-a \boldsymbol{s}+b \boldsymbol{c}):=(a+g(a, b)) \boldsymbol{c}+(b+h(a, b)) \boldsymbol{s}
$$

при всех $x \in S^{1}$ и $a, b \in \mathbb{R}$. Легко видеть, что такое определение корректно, так как в силу соотношения

$$
\left(\begin{array}{l}
u \\
p
\end{array}\right)=\left(\begin{array}{cc}
c & s \\
-s & c
\end{array}\right)\left(\begin{array}{l}
a \\
b
\end{array}\right)
$$


оно эквивалентно следующему:

$$
f(x, u, p):=(u \boldsymbol{c}-p \boldsymbol{s}+g(u \boldsymbol{c}-p \boldsymbol{s}, u \boldsymbol{s}+p \boldsymbol{c})) \boldsymbol{c}+(u \boldsymbol{s}+p \boldsymbol{c}+h(u \boldsymbol{c}-p \boldsymbol{s}, u \boldsymbol{s}+p \boldsymbol{c})) \boldsymbol{s}
$$

при всех $x, u, p$. Отметим, как из зеркальной симметрии $g, h$ в $(5.3)$ вытекает зеркальная симметрия $f$ в (5.1). Подставляя анзац

$$
u(t, x):=a(t) c+b(t) s \in E
$$

в уравнение в частных производных (1.1), мы получаем из (5.4), что такое $u$ является решением (1.1) тогда и только тогда, когда коэффициенты $a(t), b(t)$ удовлетворяют двумерному ОДУ (5.2). Это доказывает требуемую реализуемость и показывает возможность существования нестационарных периодических орбит.

Аналогичная конструкция, основанная на использовании линейной оболочки функций $\cos (n x), \sin (n x)$, показывает возможность существования нестационарных периодических орбит при наличии любого конечного числа зеркальных симметрий у уравнения в частных производных (1.1) относительно $x \in S^{1}$.

\section{6. Заключительные комментарии}

В заключительной части работы мы коротко обсудим сопутствующую проблему глобальных аттракторов для уравнения в частных производных (1.1), обобщения на квазилинейные и нелинейные уравнения, разрушение решений за конечное время, а также неявное обобщение нашей конструкции функции Ляпунова $V=\int_{0}^{1} L\left(x, u, u_{x}\right) d x$ на комплексные $p=u_{x}$.

Одна из целей использования функций Ляпунова - показать вариационный характер уравнения (1.1) на окружности и, как следствие, наличие градиентного потока. В частности, мы доказываем сходимость к равновесным состояниям. В последнее время большое внимание уделяется вопросам, посвященным глобальному аттрактору $\mathscr{A}_{f}$ (множеству ограниченных решений, которые существуют и остаются равномерно ограниченными при всех положительных и отрицательных временах), в предположении о диссипативности $f$. При наличии функции Ляпунова (1.4), (1.9), (1.10) глобальный аттрактор состоит только из равновесных состояний и их гетероклинических орбит. В противоположность этому рассмотрим $\mathrm{SO}(2)$-эквивариантный случай $f=f\left(u, u_{x}\right)$ на окружности $x \in S^{1}$, в котором не допускается функция Ляпунова. В данном случае глобальный аттрактор $\mathscr{A}_{f}$ состоит из равновесных состояний, вращающихся волн и связывающих их гетероклинических орбит (см. [12]). В [7] при изучении гетероклинических связей был использован следующий подход. Вначале все вращающиеся волны замораживались, превращаясь в результате в окружности, состоящие из неоднородных равновесных состояний. Затем, с использованием подходящих гомотопий, нелинейность $f$ симметризовалась, становясь в результате четной по $p=u_{x}$. В настоящей работе мы получаем функцию Ляпунова в явном виде для рассмотрения симметризованного случая замороженных волн. При изучении оставшихся гетероклинических орбит между равновесными состояниями в [7] основным моментом было использование свойств Штурма, 
восходящего к работе Штурма [18] 1836 г. (см. также работу [1] и приведенные там ссылки). По этой причине мы называем такие глобальные аттракторы $\mathscr{A}_{f}$ аттракторами Штурма.

Матано [11] изучал квазилинейные параболические уравнения в частных производных вида

$$
u_{t}=a\left(x, u, u_{x}\right) u_{x x}+f\left(x, u, u_{x}\right),
$$

где $a$ предполагалась равномерно положительной. Заметим, что рассуждения при выводе (2.4)-(2.11) остаются верными, если мы заменим в них $u_{x x}=u_{t}-f$ на $u_{x x}=a^{-1} u_{t}-a^{-1} f$. В частности,

$$
\dot{V}=-\int_{0}^{1} a^{-1} L_{p p}\left(u_{t}\right)^{2} d x,
$$

и нам только остается заменить $f$ на $f / a$ в (2.5)-(2.11). Аналогично, теорема 1.1 остается верной для $\mathrm{O}(2)$-эквивариантного переменного коэффициента

$$
a=a(u, p)=\bar{a}\left(u, \frac{1}{2} p^{2}\right),
$$

если мы заменим $\bar{f}$ на $\bar{f} / \bar{a}$ в (1.19)-(1.22) и подставим $L_{p p} / a$ вместо $L_{p p}$ в $(1.24)$, $(1.25)$.

Для вполне нелинейных параболических уравнений

$$
u_{t}=f\left(x, u, u_{x}, u_{x x}\right)
$$

и их эквивариантных форм существование функции Ляпунова неизвестно. При разделенных граничных условиях, по-видимому, остается надежда установить сходимость ограниченных решений к единственному состоянию равновесия с использованием свойств Штурма. Однако соответствующая техника развита пока не достаточно для того, чтобы представить здесь короткое доказательство.

Возвращаясь к $\mathrm{O}(2)$-эквивариантному полулинейному уравнению (1.1) с $f=\bar{f}\left(u, u_{x}^{2} / 2\right)$, отметим, что было бы интересно исследовать связь построенной функции Ляпунова с возможностью разрушения решений на окружности $x \in S^{1}$; см. также [6], где рассматриваются разделенные граничные условия. По существу, могут возникнуть два различных явления. В первом случае лагранжиан $L$ функции Ляпунова в (1.23) может стать неограниченно отрицательным по $u$. Во втором случае характеристики $q=q(u)$ в (1.19), (1.20) могут разрушиться уже при конечных значениях $u$, сводя на нет наше определение лагранжиана $L$ в $(1.21),(1.22)$. Было бы интересно сравнить второй из этих случаев, который может иметь место для суперквадратичных по градиенту $u_{x}$ нелинейностей $f$, с разрушением градиента, описанным в [14].

В заключение мы остановимся на любопытном комплексном феномене в нашей конструкции лагранжиана $L$ с использованием характеристик (1.19), (1.20). Для начала мы рассмотрим характеристику

$$
\frac{d}{d u} q=-\bar{f}(u, q) .
$$


Как легко видеть, там, где $q$ положительна, уравнение (6.5) имеет решением $q=q(u)$ тогда и только тогда, когда любое решение уравнения

$$
u_{x}= \pm \sqrt{2 q(u(x))}
$$

где $u(x)$ лежит в указанной области положительности $q$, является решением ОДУ равновесия

$$
0=u_{x x}+\bar{f}\left(u, \frac{1}{2} u_{x}^{2}\right)
$$

для уравнения (1.1). Для доказательства мы умножим (6.7) на $u_{x}$ и сравним результат с (6.5), воспользовавшись правилом дифференцирования сложной функции, примененным к $\frac{d}{d x} q(u(x))$. Соответственно, в каждом из случаев получим

$$
\begin{aligned}
0 & =\frac{d}{d x}\left(\frac{1}{2} u_{x}^{2}\right)+\bar{f}\left(u, \frac{1}{2} u_{x}^{2}\right) u_{x}, \\
0 & =\frac{d}{d x} q(u)+\bar{f}(u, q(u)) u_{x} .
\end{aligned}
$$

Тривиальный пример снова возникает в случае, если $\bar{f}=f(u)$ не зависит от $q$, где

$$
q=-F(u)+E,
$$

$F$ - первообразная $f$, а $E$ - энергия решения уравнения маятника $u_{x x}+f(u)=0$. При этом (6.6) принимает вид

$$
u_{x}= \pm \sqrt{2(E-F(u))} .
$$

Однако в нашем случае эволюция $\Psi^{u_{1}, u_{0}}$ характеристического уравнения (1.19), (1.20) не останавливается при $q=0$, продолжая свое развитие через отрицательное значение $q=p^{2} / 2$, что соответствует комплексному $p=u_{x}$, и снова появляясь с положительным значением в других областях фазовой плоскости $(u, q)$. В этой связи было бы очень интересно понять применимость построенных нами простых функций Ляпунова для обобщения на случай комплексных, а не только действительных значений $u$ и $u_{x}$.

\section{Список литературы}

[1] S. Angenent, "The zero set of a solution of a parabolic equation", J. Reine Angew. Math., 1988:390 (1988), 79-96.

[2] S. B. Angenent, B. Fiedler, "The dynamics of rotating waves in scalar reaction diffusion equations", Trans. Amer. Math. Soc., 307:2 (1988), 545-568.

[3] А.В. Бабин, М.И. Вишик, Аттракторы эволюиионных уравнений, Наука, М., 1989, 296 с.; англ. пер.: A. V. Babin, M. I. Vishik, Attractors of evolution equations, Stud. Math. Appl., 25, North-Holland Publishing Co., Amsterdam, 1992, x+532 pp.

[4] N. Chafee, E. F. Infante, "A bifurcation problem for a nonlinear partial differential equation of parabolic type", Appl. Anal., 4:1 (1974), 17-37.

[5] B. Fiedler, J. Mallet-Paret, "A Poincaré-Bendixson theorem for scalar reaction diffusion equations", Arch. Ration. Mech. Anal., 107:4 (1989), 325-345.

[6] B. Fiedler, H. Matano, "Global dynamics of blow-up profiles in one-dimensional reaction diffusion equations", J. Dynam. Differential Equations, 19:4 (2007), 867-893. 
[7] B. Fiedler, C. Rocha, M. Wolfrum, "Heteroclinic orbits between rotating waves of semilinear parabolic equations on the circle", J. Differential Equations, 201:1 (2004), 99-138.

[8] B. Fiedler, C. Rocha, M. Wolfrum, "Sturm global attractors for $S^{1}$-equivariant parabolic equations", Netw. Heterog. Media, 7:4 (2012), 617-659.

[9] L. F. Guidi, D. H. U. Marchetti, "Renormalization group flow of the two-dimensional hierarchical Coulomb gas", Comm. Math. Phys., 219:3 (2001), 671-702.

[10] D. Henry, Geometric theory of semilinear parabolic equations, Lecture Notes in Math., 840, Springer-Verlag, Berlin-New York, 1981, iv+348 pp.

[11] H. Matano, "Asymptotic behavior of solutions of semilinear heat equations on $S^{1}$ ", Nonlinear diffusion equations and their equilibrium states, v. II (Berkeley, CA, 1986), Math. Sci. Res. Inst. Publ., 13, eds. W.-M. Ni, L. A. Peletier, J. Serrin, Springer, New York, 1988, 139-162.

[12] H. Matano, K.-I. Nakamura, "The global attractor of semilinear parabolic equations on $S^{1 ",}$ Discrete Contin. Dyn. Systems, 3:1 (1997), 1-24.

[13] X. Mora, "Semilinear parabolic problems define semiflows on $C^{k}$ spaces", Trans. Amer. Math. Soc., 278:1 (1983), 21-55.

[14] О. А. Олейник, С. Н. Кружков, "Квазилинейные параболические уравнения второго порядка со многими независимыми переменными", УМН, 16:5(101) (1961), 115-155; англ. пер.: O. A. Oleinik, S. N. Kruzhkov, "Quasi-linear second-order parabolic equations with many independent variables", Russian Math. Surveys, 16:5 (1961), 105-146.

[15] A. Pazy, Semigroups of linear operators and applications to partial differential equations, Appl. Math. Sci., 44, Springer-Verlag, New York, 1983, viii+279 pp.

[16] C. Ragazzo, "Scalar autonomous second order ordinary differential equations", Qual. Theory Dyn. Syst., 11:2 (2012), 277-415.

[17] B. Sandstede, B. Fiedler, "Dynamics of periodically forced parabolic equations on the circle", Ergodic Theory Dynam. Systems, 12:3 (1992), 559-571.

[18] C. Sturm, "Sur une classe d'équations à différences partielles", J. Math. Pures Appl., 1 (1836), 373-444.

[19] Т.И. Зеленяк, "О стабилизации решений краевых задач для параболического уравнения второго порядка с одной пространственной переменной", Дифферени. уравнения, 4:1 (1968), 34-45; англ. пер.: Т. I. Zelenyak, "Stabilization of solutions of boundary value problems for a second-order parabolic equation with one space variable", Differ. Equ., 4 (1972), 17-22.

[20] T. I. Zelenyak, M. M. Lavrentiev Jr., M. P. Vishnevskii, Qualitative theory of parabolic equations. Part 1, VSP, Utrecht, 1997, ii+417 pp.

\section{Бернольд Фидлер}

Поступила в редакцию

(Bernold Fiedler)

25.10 .2013

Institut für Mathematik, Freie Universität Berlin,

Berlin, Germany

E-mail: fiedler@mi.fu-berlin.de

\section{Клодоальдо Гротта-Рагаццо}

(Clodoaldo Grotta-Ragazzo)

Instituto de Matemática e Estatística,

Universidade de São Paulo,

São Paulo, Brazil

E-mail: ragazzo@ime.usp.br

\section{Карлош Роша}

(Carlos Rocha)

Instituto Superior Técnico,

Lisboa, Portugal

E-mail: crocha@math.ist.utl.pt 